\title{
Comparative clinical analysis of fever in tumor patients, normal patients, and those infected with new coronavirus pneumonia
}

\author{
Minting Ma ${ }^{1}$, Junyan Wang ${ }^{1}$, Fan Zhang ${ }^{1}$, Lei Hong ${ }^{1}$, Lei Zhao ${ }^{2}$, Bo Hu ${ }^{3}$, Zhaojun Wu ${ }^{1}$, Suju Wei ${ }^{1}$, \\ Yu Xu' ${ }^{1}$, Peng Guo ${ }^{4}$, Bin Guo ${ }^{5}$ \\ ${ }^{1}$ Department of Oncology, The Fourth Hospital of Hebei Medical University, Shijiazhuang, China; ${ }^{2}$ Department of Internal Medicine, Shijiazhuang \\ Fifth Hospital, Shijiazhuang, China; ${ }^{3}$ Department of Oncology, Hebei Chest Hospital, Shijiazhuang, China; ${ }^{4}$ Department of Orthopedics, The \\ Fourth Hospital of Hebei Medical University, Shijiazhuang, China; ${ }^{5}$ Department of Thoracic surgery, The Fourth Hospital of Hebei Medical \\ University, Shijiazhuang, China \\ Contributions: (I) Conception and design: M Ma, B Guo; (II) Administrative support: S Wei; (III) Provision of study materials or patients: L Zhao, \\ B Hu, Z Wu; (IV) Collection and assembly of data: J Wang, F Zhang, Y Xu; (V) Data analysis and interpretation: L Hong, P Guo; (VI) Manuscript \\ writing: All authors; (VII) Final approval of manuscript: All authors. \\ Correspondence to: Bin Guo. Department of Thoracic surgery, The Fourth Hospital of Hebei Medical University, 12 Jiankang Road, Shijiazhuang \\ 050000, China. Email: guowowo2017@sina.com.
}

Background Under the current epidemic of the coronavirus disease of 2019 (COVID-19), there is a need to distinguish the differences between the laboratory examinations of COVID-19-infected patients, tumor patients with fever, and those with normal fever patients. We aimed to investigate the temperature of tumor patients with different tumor burdens, stages, and cancer types.

Methods: We recruited 3 groups of patients to this study: fever patients with malignant tumors, ordinary fever patients, and confirmed cases of COVID-19, with 31, 55, and 28 cases in each group, respectively.

Results: The levels of leukocytes and neutrophils were the highest among non-tumor patients, and the count of COVID-19 was the lowest, with a $\mathrm{P}$ value of 0.000 . Among the leukocytosis group, non-tumor patients had the highest proportion (43.6\%), while that of COVID-19 was only $3.6 \%(\mathrm{P}=0.000)$. Similarly, there were significant differences in the grading of neutrophils, where most of the infected patients were in the normal group and the $\mathrm{P}$ value was 0.000 . The lymphocyte count of the tumor group was significantly reduced, with an average of $(0.97 \pm 0.66) \times 10^{9} / \mathrm{L}(\mathrm{P}=0.004)$. In the lymphocyte grades, most of the infected patients were the normal group $(71.4 \%)$, while tumor patients in the lymphocytopenia group accounted for $63.1 \%(\mathrm{P}=0.006)$. There were also significant differences in the neutrophil to lymphocyte ratio (NLR) $(\mathrm{P}=0.006)$. There was a significant difference in temperature between different tumor burden groups $(\mathrm{P}=0.014)$.

Conclusions: The normal fever group had the highest count of leukocyte and neutrophils, whereas the infected group had the lowest relative count. The NLR was the lowest in the infected group. The NLR was higher in the bigger tumor load group.

Keywords: Fever; malignant tumor; 2019 novel coronavirus epidemic situation (2019-nCoV epidemic situation); blood laboratory index

Submitted Nov 23, 2021. Accepted for publication Feb 14, 2022.

doi: 10.21037/apm-21-3665

View this article at: https://dx.doi.org/10.21037/apm-21-3665 


\section{Introduction}

The influenza pandemic of 1918 incurred the largest death toll in human history. Fatalities exceeded 50 million, which is equivalent to 200 million of the global population today. The emergence of coronaviruses has involved different trajectories, but the principle is similar: severe acute respiratory syndrome (SARS), the Middle East respiratory syndrome (MERS) and coronavirus disease of 2019 (COVID-19) have all apparently originated from a bat virus (1). In late December 2019, an unexplained viral pneumonia appeared in Wuhan, China. After a few weeks, this unknown viral pneumonia broke out rapidly, and the number of infections surged. Sequencing of airway epithelial cell samples from patients with this type of pneumonia isolated a new coronavirus named the 2019 novel coronavirus (2019-nCoV) (2). The main mode of transmission of this virus is droplets and close contact transmission. In addition, studies have found that the virus can be excreted through feces and urine, but it is not clear whether it can be transmitted through excrement (3). Among the first batch of 425 patients diagnosed with new coronavirus pneumonia, the median age was 59 years and $56 \%$ were men. The average incubation period is 5.2 days. In the early stages, the epidemic doubled every 7.4 days (4). The newly discovered zoonotic coronavirus severe acute respiratory syndrome coronavirus-2 (SARS-CoV-2) has the characteristics of rapid human-to-human transmission.

Cancer patients frequently visit the hospital for treatment and review, and they may be hypoimmune due to potential malignant tumors or anti-cancer treatments, thus having a higher risk of infection. The risk of SARS-CoV-2 infection for cancer patients seems to be double that of the general population (5). The innovation of this paper is to identify patients with fever as the main symptom, especially in cancer hospitals. There are many people with fever in cancer patients. How to diagnose the cause of fever is of great significance to doctors in cancer hospitals.

The globally explosive growth, mortality rate, and the current epidemic prevention situation are still severe. The outbreak of the epidemic has brought great changes to the normal life and order of medical treatment in China, especially for patients with malignant tumors, where most patients have been delayed access to anti-tumor treatment in the hospital. In addition, due to the strengthening of epidemic prevention, special attention is being paid to the treatment of patients with fever. Nucleic acid testing has a certain waiting time, and has been associated with false negative results. In some tumor patients, fever has a specific cause, such as tumor-related factors, antitumor drugs, and so on. Therefore, question shave arisen regarding the initial differentiation and diagnosis of fever using existing clinicpathological and laboratory tests for tumor patients with fever, and how to differentiate between tumor patients, and non-tumor fever patients, and COVID-19 patients. To address these questions, we collected and diagnosed febrile tumor patients and compared them with the clinical data and laboratory test results of new coronavirus pneumonia and the statistics of non-tumor patients who visited fever clinics and had been excluded for new coronavirus pneumonia through imaging, laboratory examination, or nucleic acid screening. We aimed to determine the differences between the 3 groups of fever patients during this $2019-\mathrm{nCoV}$ epidemic, to guide and learn from the preliminary assessment of febrile tumor patients.

We present the following article in accordance with the STROBE reporting checklist (available at https://apm. amegroups.com/article/view/10.21037/apm-21-3665/rc).

\section{Methods}

All procedures performed in this study involving human participants were in accordance with the Declaration of Helsinki (as revised in 2013). This research was approved by the ethics committee of The Fourth Hospital of Hebei Medical University (Ethics Review Number: 2022KS001). Individual consent for this retrospective analysis was waived. Malignant tumor patients who had been admitted to the Department of Oncology, The Fourth Hospital of Hebei Medical University from 31 January 2020 to 25 April 2020 were recruited to the study. During the period, the patients had exhibited fever at least once. According to the new coronavirus pneumonia prevention and control process, participants had undergone relevant inspections and those with new coronavirus pneumonia were excluded. Finally, 31 people were selected for the febrile tumor group. The clinical data of these patients included gender, age, cancer type, stage, and so on. After the patient developed a fever, the relevant laboratory test results were applied, including blood routine, C-reactive protein (or rapid CRP), procalcitonin (PCT), blood culture, and so on. The normal value of neutrophil count is $(1.8-6.3) \times 10^{9} / \mathrm{L}$, the normal value of leukocyte count is $(3.5-9.5) \times 10^{9} / \mathrm{L}$, the normal value of lymphocyte count is $(1.1-3.2) \times 10^{9} / \mathrm{L}$, the normal value of rapid CRP is $0-4 \mathrm{mg} / \mathrm{L}$, the normal value of CRP is $0-6 \mathrm{mg} / \mathrm{L}$, and that of PCT is $<0.046 \mathrm{ng} / \mathrm{L}$. The maximum 
Table 1 The basic characteristics of the 3 groups

\begin{tabular}{lccc}
\hline Characteristics & Tumor group, $\mathrm{n}(\%)$ & Normal group, $\mathrm{n}(\%)$ & Infected group, $\mathrm{n}(\%)$ \\
\hline Gender & $13(41.9)$ & $26(47.3)$ & $16(57.1)$ \\
Male & $18(58.1)$ & $29(52.7)$ & $12(42.9)$ \\
Female & & & \\
Age classification & $4(12.9)$ & $37(67.3)$ & $13(46.4)$ \\
$\leq 40$ years & $17(54.8)$ & $11(20.0)$ & $5(17.9)$ \\
$>40$ and $\leq 60$ years & $10(32.3)$ & $7(12.7)$ & $10(35.7)$ \\
$>60$ years & &
\end{tabular}

body temperature, record chest computed tomography (CT) exudation and other combined infections, tumor burden, and so on, were recorded. Another group of patients who had been treated at the fever clinic of our hospital and been diagnosed without tumor between 20 February 2020 and 31 March 2020 were recruited to the study. All patients in the fever clinic were screened for non-new coronavirus pneumonia, and a total of 55 patients were collected who received routine examination methods such as laboratory testing, chest CT, and nucleic acid testing (32 patients with nucleic acid testing). The last group included patients diagnosed with new coronavirus pneumonia by nucleic acid testing, collected the general information and laboratory examinations at Shijiazhuang Fifth Hospital and Hebei Chest Hospital from February 2020 to July 2020.

\section{Statistical analysis}

Data were analyzed using the software SPSS 23.0 (IBM Corp., Chicago, IL, USA). The count data was expressed as a percentage, frequency was used for descriptive analysis, and the difference was compared with the cross-tab $\chi^{2}$ test. The measurement data were statistically described by the mean \pm standard deviation, the difference between 3 groups was compared by one-way analysis of variance (ANOVA) method, and the difference was statistically significant when $\mathrm{P}<0.05$.

\section{Results}

\section{The basic characteristics of the 3 groups (Table 1)}

A total of 31 cancer patients were included, including 13 males and 18 females. There were 55 patients in the normal group, including 26 males and 29 females. There were 28 patients with new coronavirus pneumonia infection group, including 16 males and 12 females. There was no difference in gender between the 3 groups. The average age of the tumor group was $54.87 \pm 11.168$ years, $37.35 \pm 18.458$ years in the normal group, and $46.07 \pm 19.931$ years in the infected group. After single factor ANOVA, $\mathrm{P}=0.000$. The proportion of patients over 60 years old was $32.3 \%$ in the cancer group, $35.7 \%$ in the infected group, while only $12.7 \%$ in the normal group, by $\chi^{2}$ square test, $\mathrm{P}=0.000$. All confirmed COVID-19 patients had pneumonia confirmed by imaging, and the proportion of normal group and cancer group indicated concurrent other pathogenic infections by chest CT examination was respectively. $22.6 \%$ and $16.4 \%$ respectively, $\mathrm{P}=0.477$.

\section{Comparison number of laboratory examination (Table 2)}

According to statistics, the average \pm standard deviation of the white blood cell (WBC) counts of the tumor group, normal group, and infected group was $(7.94 \pm 4.63) \times 10^{9} / \mathrm{L}$, $(10.38 \pm 5.30) \times 10^{9} / \mathrm{L}$, and $(5.12 \pm 1.62) \times 10^{9} / \mathrm{L}$, respectively. The normal group had the highest WBC, followed by the tumor group, and the $\mathrm{WBC}$ count of patients with COVID-19 was the lowest, $\mathrm{P}=0.000$. Similarly, the highest neutrophil count among the 3 groups was $(7.28 \pm 4.47) \times 10^{9} / \mathrm{L}$ in the normal group, followed by $(6.45 \pm 4.30) \times 10^{9} / \mathrm{L}$ in the tumor group, and the lowest was the COVID-19 group at $(3.20 \pm 1.59) \times 10^{9} / \mathrm{L}, \mathrm{P}=0.000$. The lymphocyte count in the normal group was $(1.79 \pm 1.32) \times 10^{9} / \mathrm{L}$, followed by $(1.55 \pm 0.77) \times 10^{9} / \mathrm{L}$ in infected group, and the lowest was the tumor group at $(0.97 \pm 0.66) \times 10^{9} / \mathrm{L}, \mathrm{P}=0.004$. The NLR is the ratio of neutrophil to lymphocytes: the highest value of NLR was $13.42 \pm 2.41$ in the tumor group, and the lowest value was in the infected group at $3.17 \pm 0.60, \mathrm{P}=0.006$. 
Table 2 Comparison number of laboratory examination results between the 3 groups

\begin{tabular}{lcccc}
\hline Variable & Tumor group, mean \pm SD & Normal group, mean \pm SD & Infected group, mean \pm SD & P value \\
\hline Leukocyte $\left(\times 10^{9} / \mathrm{L}\right)$ & $7.94 \pm 4.63$ & $10.38 \pm 5.30$ & $5.12 \pm 1.62$ & 0.000 \\
Neutrophils $\left(\times 10^{9} / \mathrm{L}\right)$ & $6.45 \pm 4.30$ & $7.28 \pm 4.47$ & $3.20 \pm 1.59$ & 0.000 \\
Lymphocyte $\left(\times 10^{9} / \mathrm{L}\right)$ & $0.97 \pm 0.66$ & $1.79 \pm 1.32$ & $1.55 \pm 0.77$ & 0.004 \\
NLR & $13.42 \pm 2.41$ & $7.81 \pm 1.05$ & $3.17 \pm 0.60$ & 0.006 \\
\hline
\end{tabular}

SD, standard deviation; NLR, neutrophil to lymphocyte ratio.

Table 3 Comparison of laboratory examination results levels between the 3 groups

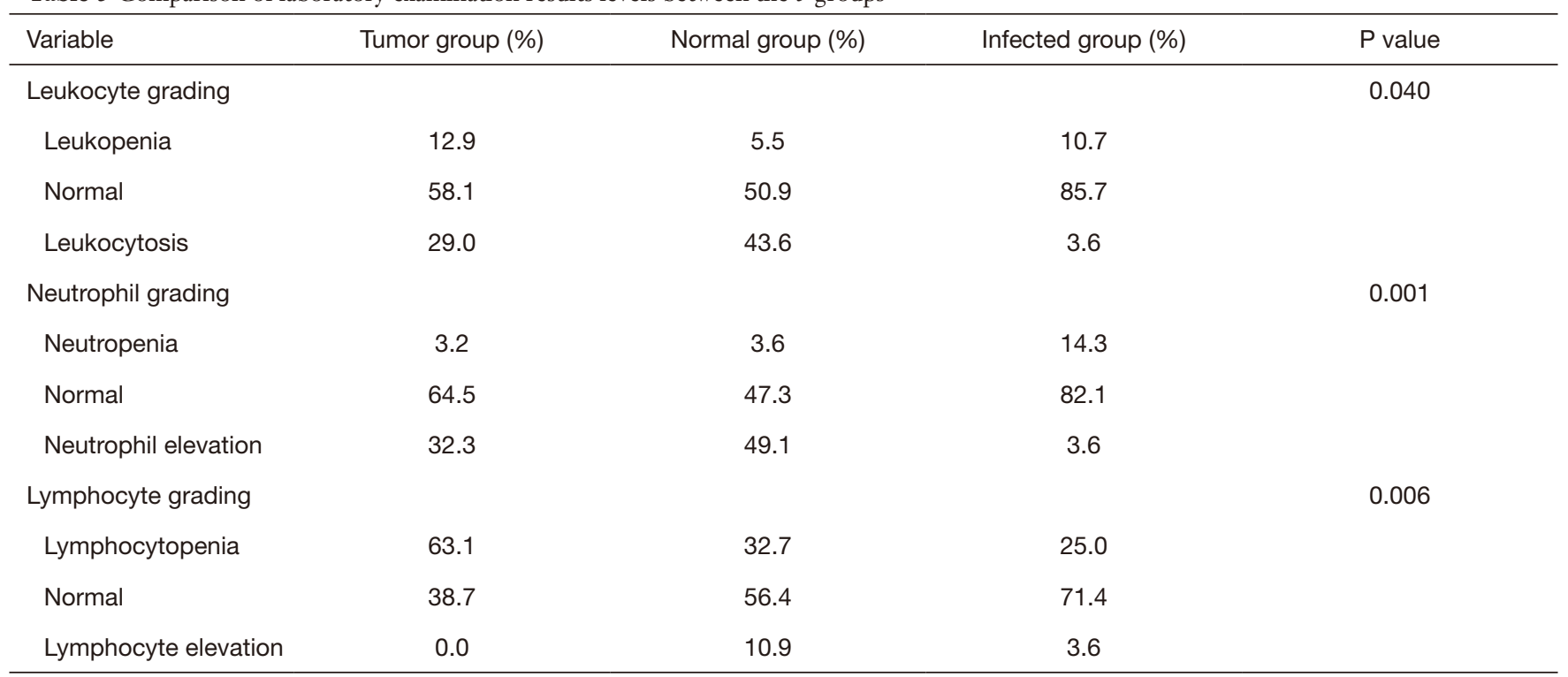

\section{Comparison levels of laboratory examination (Table 3)}

According to the laboratory test results, the normal value reference range of leukocyte count, neutrophil count, and lymphocyte count were set to 3 levels: lower than normal, normal, and higher than the normal. In the infected group, $85.7 \%$ of cases were in normal leukocyte range, $10.7 \%$ had leukopenia, and only $3.6 \%$ had leukocytosis. The proportion of leukocytosis of patients in the tumor group and the normal group was higher, than that of the infected group, at $29 \%$ and $43.6 \%$, respectively. The leukocytes level of the 3 groups was $\mathrm{P}=0.04$. The proportion of normal neutrophils of tumor patients was $64.5 \%$, and $32.3 \%$ had elevated neutrophils. The proportions of normal neutrophils and neutrophil elevation in the normal group were $47.3 \%$ and $49.1 \%$, respectively, while the proportions of normal neutrophils and neutrophil elevation in the infected group were $82.1 \%$ and $3.6 \%, P=0.001$. In the lymphocyte level classification, $63.1 \%$ of tumor patients had lymphocytopenia, $38.7 \%$ were in the normal level, $71.4 \%$ of infected group were in the normal level, and $25.0 \%$ had lymphocytopenia, $\mathrm{P}=0.006$.

\section{Febrile tumor patient characteristics}

A total of 31 cancer patients were enrolled. According to the type of cancer, they were divided into respiratory system tumors, digestive system tumors, breast or gynecological system tumors, and others [cancer of unknown primary (CUP) or kidney cancer], of which the proportions were $12.9 \%, 64.5 \%, 12.9 \%$, and $9.7 \%$, respectively. All cases measured the sum of the diameters of the larger tumors in the various organs of the current system (for multiple lesions in one organ, the largest diameter was selected, and bone metastases were not counted). Taking $10 \mathrm{~cm}$ as the boundary, they were divided into a $\leq 10 \mathrm{~cm}$ group and 
$>10 \mathrm{~cm}$ group, with 25 cases and 6 cases, respectively. All cancer patients were divided into stage II, stage III, and stage IV, for which the proportions were $3.2 \%, 6.5 \%$, and $90.3 \%$, respectively. Considering that the proportion of stage II and stage III patients was relatively low, they were divided into a non-advanced tumor group and advanced tumor group, which accounted for $9.7 \%$ and $90.3 \%$ respectively. For all hospitalized patients with fever, clinicians ruled out new coronavirus pneumonia by applying the relevant tests, examinations, and consultations in relevant departments. At the same time, they also diagnosed and differentially diagnosed the cause of fever. The final analysis was 13 cases related to oncology drugs. The common drugs included paclitaxel $(n=5)$, oxaliplatin $(n=2)$, irinotecan $(\mathrm{n}=2)$, and immunosuppressants $(\mathrm{n}=2)$.

The highest body temperature single-factor ANOVA or independent sample $t$-test was performed according to different cancer types, burden stratifications, and stage. The statistical results showed that in different cancer types: there was no significant statistical difference in temperature of these groups of patients, $\mathrm{P}=0.305$. There was no significant difference between the non-advanced tumor group and the advanced tumor group, $\mathrm{P}=0.213$. However, there were significant differences in body temperature in different tumor burdens, $\mathrm{P}=0.014$, the mean \pm standard deviation of the body temperature of the 2 groups were $38.364 \pm 0.6217$ and $37.683 \pm 0.1941{ }^{\circ} \mathrm{C}$, respectively. The body temperature of the tumor burden $\leq 10 \mathrm{~cm}$ group was significantly higher.

\section{Discussion}

Fever is a common symptom of COVID-19 infection, presenting in $98 \%$ of new cases (3). A population-based study confirmed that fever of unknown origin is related to cancer. This study linked the Danish National Patient Registry with the National Cancer Registry and evaluated the first time after admission for fever of unknown origin between 1977 and 1997. For the cancer risk of 43,205 patients discharged from the Danish hospital, we compared the cancer incidence with the expected cancer incidence. The median follow-up was 6.3 years, and the results showed that in the first year of follow-up, 399 cases were diagnosed with cancer, and the proportion of diagnosed cancer within 1 year after discharge from hospital with unexplained fever was slightly higher than that of the control group (6). During the epidemic, some cancer patients will have fever, which can be a cause of panic or anxiety. Some patients even have antitumor treatments temporarily suspended due to epidemic prevention and control. However, not all patients with fever have COVID-19. There are many reasons for fever, such as infectious diseases, connective tissue diseases, and neoplastic diseases (7). Clinical and laboratory examinations of different causes of fever also have some identifiable characteristics. Meta-analysis can be used to identify the common characteristics of patients with COVID-19. A meta-analysis included a total of 55 retrospective studies involving 8,697 COVID-19 patients and revealed that the main manifestation was fever (78.4\%), most patients had normal WBC count (64.7\%), and other findings included leukopenia (23.5\%), leukocytosis (9.9\%), and lymphopenia (47.6\%) (8). Especially in critically ill patients, lymphocytopenia is more obvious, so it is speculated that this virus destroys the body's defense system, leading to a severe inflammatory storm, which causes multiple organ failure (9). In this study, $25.0 \%$ of the patients in the infected group had lymphocytopenia. Compared with this meta-analysis, the percentage of lymphocytopenia was lower. By analyzing the reasons, all the cases with infection in this study were the early blood indexes after the diagnosis of COVID-19, and most of the patients were mild. The percentages of normal leukocytes and neutrophils were $85.7 \%$ and $82.1 \%$, respectively. A multicenter retrospective study included new coronal infection. According to CT and clinical characteristics, the patients were divided into stable and progressive groups. The lymphocyte count of stable group was $1.1 \times 10^{9} / \mathrm{L}$, which was significantly higher than that of disease progression group. The NLR of the 2 groups was 2.5 and 4.8 , and the disease progression group was significantly higher. In this study, the lymphocyte count of infected patients was $1.55 \times 10^{9} / \mathrm{L}$, and the NLR was 3.17 . Considering that the data in this study were drawn from the beginning of infection and the disease was earlier, the lymphoid count was relatively normal, and there was no significant decrease, and the NLR value was not significantly increased; however, this study confirmed that NRL in the infected group was significantly lower than that the other groups (10).

According to the final analysis, 13 cases $(41.9 \%)$ had fever related to anti-tumor drugs, including paclitaxel $(n=5)$, oxaliplatin $(\mathrm{n}=2)$, irinotecan $(\mathrm{n}=2)$ and immunosuppressant $(n=2)$. A retrospective analysis of 1,016 consecutive cancer cycles was conducted to analyze the characteristics of fever after cancer chemotherapy. The results showed that fever accounted for $36 \%$ of all treatment cycles, and the most common was 4,3 , and 12 days after treatment (11). The types of fever caused by antineoplastic drugs can be divided into several types, such as neutropenic fever and 
drug-related fever. All the drug-related fever recorded in this study occurred in 1-2 days after the application of chemotherapy drugs. Paclitaxel was the most common drug related to fever in this study. At present, tumor therapy has entered the immune era, and the efficacy of immunotherapy is being explored in different kinds of cancer. However, the mystery of immunotherapy has not been fully revealed in terms of treatment mechanism, biological prediction, and adverse reactions. In this study, 2 patients developed transient fever after immunotherapy, and the sample size was limited. Therefore, the mechanism of fever caused by immunotherapy still needs to be further explored. Natural killer T (NKT) is a CD1d restricted lymphoid cell characterized by invariant $\mathrm{T}$ cell receptors, which are co-expressed in NKT by pairing with $\mathrm{V} \alpha 24^{+} \mathrm{V} \beta 11^{+}$chain. These cells rapidly produce a large number of cytokines [such as interferon (IFN)- $\gamma$ and interleukin (IL)-4] to regulate other cells of the immune system, such as $\mathrm{T}$ cells, NK cells, and dendritic cells. This study found that the number of $\mathrm{V} \alpha 24^{+} \mathrm{V} \beta 11^{+} \mathrm{NKT}$ cells in 120 cancer patients was about $50 \%$ lower than that in healthy control group. The V $\alpha 24^{+} \mathrm{V} \beta 11^{+} \mathrm{NKT}$ of cancer patients was not affected by tumor load, but it may reflect tumor development, for example, by hindering effective tumor immune surveillance (12). The results of this study showed that the lymphatic count of the tumor group was significantly decreased, $\mathrm{P}=0.000$, which may be related to the immune system suppression of tumor patients, and there was no statistical difference in lymph count among different tumor loads, which was also consistent with the results of this basic experimental study. Another study confirmed that tumor load significantly inhibited the differentiation of $\mathrm{T} 1$ and $\mathrm{T} 2$ cells in colorectal cancer patients, and it could be an important factor in the development of immunosuppression. After the tumor burden was eliminated, the percentage of $\mathrm{T} 1$ and $\mathrm{T} 2$ increased in a short time, and the immune function was improved (13).

There are many reasons for fever in patients with malignant tumor, which are different from other non-tumor fever, such as neutrophil deficiency fever, patients with large tumor load, or rapid tumor progression or terminal stage may have different degrees of cancerous fever (14), and the causes of antineoplastic drugs such as chemotherapy, immunotherapy, and bisphosphonates drug induced fever. At present, when novel coronavirus pneumonia is prevalent, patients should also be diagnosed with their type of fever at the time of febrile manifestation. At present, the epidemic prevention and control is entering the normalization stage, and a history of fever is required. In the case of patients with fever, we need to improve blood routine and chest CT first. If NLR level is high and chest CT shows typical viral pneumonia, it is necessary to consider full epidemiological investigation and multiple nucleic acid tests. At the same time, we must ask patients about recent anti-tumor treatment, and have a comprehensive judgement of the overall patient. Throat swab test should be performed when necessary. Novel coronavirus pneumonia can be delayed by adjuvant chemotherapy or surgical treatment. If the new coronavirus pneumonia is excluded, the tumor will continue to be treated throughout the COVID-19 epidemic period, so as not to delay the treatment and cause recurrence and metastasis of cancer (15).

\section{Acknowledgments}

Funding: None.

\section{Footnote}

Reporting Checklist: The authors have completed the STROBE reporting checklist. Available at https://apm. amegroups.com/article/view/10.21037/apm-21-3665/rc

Data Sharing Statement: Available at https://apm.amegroups. com/article/view/10.21037/apm-21-3665/dss

Conflicts of Interest: All authors have completed the ICMJE uniform disclosure form (available at https://apm. amegroups.com/article/view/10.21037/apm-21-3665/coif). The authors have no conflicts of interest to declare.

Ethical Statement: The authors are accountable for all aspects of the work in ensuring that questions related to the accuracy or integrity of any part of the work are appropriately investigated and resolved. All procedures performed in this study involving human participants were in accordance with the Declaration of Helsinki (as revised in 2013). This research was approved by the ethics committee of The Fourth Hospital of Hebei Medical University (Ethics Review Number: 2022KS001). Individual consent for this retrospective analysis was waived.

Open Access Statement: This is an Open Access article distributed in accordance with the Creative Commons Attribution-NonCommercial-NoDerivs 4.0 International License (CC BY-NC-ND 4.0), which permits the non- 
commercial replication and distribution of the article with the strict proviso that no changes or edits are made and the original work is properly cited (including links to both the formal publication through the relevant DOI and the license). See: https://creativecommons.org/licenses/by-nc-nd/4.0/.

\section{References}

1. Morens DM, Daszak P, Taubenberger JK. Escaping Pandora's Box - Another Novel Coronavirus. N Engl J Med 2020;382:1293-5.

2. Zhu N, Zhang D, Wang W, et al. A Novel Coronavirus from Patients with Pneumonia in China, 2019. N Engl J Med 2020;382:727-33.

3. Special Expert Group for Control of the Epidemic of Novel Coronavirus Pneumonia of the Chinese Preventive Medicine Association. An update on the epidemiological characteristics of novel coronavirus pneumonia (COVID-19). Zhonghua Liu Xing Bing Xue Za Zhi 2020;41:139-44.

4. Li Q, Guan X, Wu P, et al. Early Transmission Dynamics in Wuhan, China, of Novel Coronavirus-Infected Pneumonia. N Engl J Med 2020;382:1199-207.

5. Al-Shamsi HO, Alhazzani W, Alhuraiji A, et al. A Practical Approach to the Management of Cancer Patients During the Novel Coronavirus Disease 2019 (COVID-19) Pandemic: An International Collaborative Group. Oncologist 2020;25:e936-45.

6. Sørensen HT, Mellemkjaer L, Skriver MV, et al. Fever of unknown origin and cancer: a population-based study. Lancet Oncol 2005;6:851-5.

7. Ji WJ, Yan XB. Epidemiological and clinical characteristics of fever of unknown origin. Chinese Journal of Nosocomiology 2019;29:3681-6.

8. Zhu J, Zhong Z, Ji P, et al. Clinicopathological characteristics of 8697 patients with COVID-19 in China: a meta-analysis. Fam Med Community Health 2020;8:e00406.

9. Li D, Wang M, He B, et al. Laboratory test analysis of sixty-two COVID-19 patients. Medical Journal of Wuhan University 2020;41:861-5.

10. Feng $Z, Y u$, Yao $S$, et al. Early prediction of disease progression in COVID-19 pneumonia patients with chest CT and clinical characteristics. Nat Commun 2020;11:4968.

11. Ogawara D, Fukuda M, Ueno S, et al. Drug fever after cancer chemotherapy is most commonly observed on posttreatment days 3 and 4 . Support Care Cancer 2016;24:615-9.

12. Molling JW, Kölgen W, van der Vliet HJ, et al. Peripheral blood IFN-gamma-secreting Valpha24+Vbeta11+ NKT cell numbers are decreased in cancer patients independent of tumor type or tumor load. Int J Cancer 2005;116:87-93.

13. Cui M, Wang S, Ye YJ, et al. Effect of tumor burden on differentiation of $T$ lymphocytes in the peripheral blood of patients with colorectal cancer. Zhonghua Yi Xue Za Zhi 2007;87:16-9.

14. Pasikhova Y, Ludlow S, Baluch A. Fever in Patients With Cancer. Cancer Control 2017;24:193-7.

15. Liang W, Guan W, Chen R, et al. Cancer patients in SARS-CoV-2 infection: a nationwide analysis in China. Lancet Oncol 2020;21:335-7.

(English Language Editor: J. Jones)

Cite this article as: Ma M, Wang J, Zhang F, Hong L, Zhao L, Hu B, Wu Z, Wei S, Xu Y, Guo P, Guo B. Comparative clinical analysis of fever in tumor patients, normal patients, and those infected with new coronavirus pneumonia. Ann Palliat Med 2022;11(2):544-550. doi: 10.21037/apm-21-3665 